\title{
Pratiques agricoles et perceptions paysannes des impacts environnementaux de la cotonculture dans la province de la KOMPIENGA (Burkina Faso)
}

\author{
Alain P.K. Gomgnimbou ${ }^{1 *}$, PaulW. Savadogo ${ }^{2}$, Aimé J. Nianogo ${ }^{3}$ \& Jeanne Millogo-RasolodimbY4. \\ 'Institut de l'Environnement et de Recherches Agricoles (INERA). Station de Farako-Bâ. 01 BP 910 Bobo-Dioulasso. \\ ${ }^{2}$ Institut de l'Environnement et de Recherches Agricoles (INERA). Station de Kamboinsé. 01 BP 476 Ouagadougou. \\ ${ }^{3}$ Union Mondiale pour la Conservation de la Nature et des ressources (UICN). Bureau Régional Afrique de l'Ouest et du Centre. \\ Ouagadougou/ Burkina Faso. \\ ${ }^{4}$ Université de Ouagadougou. Laboratoire de biologie et écologie végétales. UFR/SVT. \\ *Auteur pour les correspondances (Email : gpkalain@yahoo.fr) \\ Reçu le 10-12-2010, accepté le 21-06-2010
}

\begin{abstract}
Résumé
La dégradation des écosystèmes et d'une manière générale de l'environnement au Burkina Faso est liée aux facteurs anthropiques, notamment les activités agricoles. L'objectif de cette étude est de mettre en exergue les pratiques agricoles, les perceptions paysannes des impacts environnementaux et les risques de dégradation des ressources naturelles dans la zone cotonnière de l'Est (Province de la Kompienga). Par le biais (i) d'une enquête auprès d'un échantillon de 60 Unités de Production Cotonnière (UPC), (ii) des interviews semi structurées avec des maraîchers (12), des apiculteurs (15), des pêcheurs (29), des éleveurs (54) et des membres de comités villageois de gestion des forêts (23), (iii) et des investigations sur les pratiques agricoles, l'étude a été conduite. Les résultats ont révélé une pression foncière dans la zone avec pour corollaire un abandon des techniques de conservation et de restauration des sols. L'usage des fertilisants organiques est peu répandu. L'étude a révélé que 8 types de rotations culturales sont pratiquées dans la zone. Le type de rotation culturale coton-céréales-coton est pratiqué par $63 \%$ des cotonculteurs. Les perceptions paysannes sur la dégradation des ressources végétales, les risques encourus par l'écologie apicole ainsi que des effets négatifs des fertilisants chimiques sur l'eau et les sols sont diversement appréciés. Face à cette situation, il est urgent pour les producteurs de coton d'utiliser les engrais organiques et d'adopter des techniques de conservation et de restauration des sols pour préserver l'exploitation durable des ressources naturelles de la zone.
\end{abstract}

Mots-clés : Culture de coton; Pratiques agricoles; Pression foncière; Risque de dégradation de l'environnement; Burkina Faso.

\section{Abstract \\ Agricultural practices and peasant pereceptions of environmental problems in the province of KOMPIENGA (Burkina faso).}

Ecosystem degradation and a general an environment in Burkina Faso is linked to anthropogenic factors such as agricultural activities. The aim of this study was to highlight the agricultural practices, the farmer's perceptions of environmental impacts and the risks of natural resources degradation in the cotton belt of the East (Kompienga's Province). Through (i) a survey of a sample of 60 Cotton Production Units (UPC), (ii) semi-structured interviews with gardeners (12), bee (15), fishermen (29), breeders (54) and members of the village committee of forest management (23), (iii) and investigations on agricultural practices have been conducted. The results showed an increased pressure on land with a concomitant abandonment of land conservation and restoration techniques. The use of organic fertilizers is not widespread. The study revealed that 8 types of crop rotations are practiced in the area. The type cotton-cerealcotton type is practiced by $63 \%$ of cotton growers. Farmers' perceptions on the degradation of plant resources, the risks incurred by the bees ecology and the negative effects of chemical fertilizer on water and land are valued differently. In this context it is urgent for cotton farmers to use organic fertilizers and adopting land conservation and restoration techniques for lead to the sustainable management of natural resources in the area.

Key words: Cotton culture; Agricultural Practices; Land pressure; Risk of Environment degradation; Burkina Faso. 


\section{Introduction}

Le coton est une culture stratégique au sein de certaines exploitations familiales agricoles des pays de l'Afrique de l'Ouest, de l'Est et du Centre. On estime que 2 à 3 millions de ménages d'Afrique de l'Ouest et du Centre cultivent le coton sur des champs d'une superficie moyenne de 1 ha (Hussein et al., 2005). L'Afrique de l'ouest se situe au troisième rang mondial des exportateurs (près d'un million de tonnes en moyenne sur la période de 2000/01 à 2004/05, soit 13\%), derrière les EtatsUnis (2,5 millions de tonnes, soit $37 \%)$ et l'Asie centrale $(1,2$ million de tonnes, soit $17 \%)$ (CEDEAO-CSAO/OCDE, 2006).

Le coton est la principale culture de rente du Burkina Faso. La production record du pays a été de 712707 tonnes en 2005 (MAHRH, 2006). Cette spéculation a procuré près de $70 \%$ de recettes d'exportation agricole et est considérée comme un choix stratégique de développement du pays (Ton, 2006).

L'extension et l'accroissement de la production cotonnière dans la région de l'Est du Burkina Faso ont été confortés par «le plan de relance de la culture cotonnière» adopté en février 1996 par le gouvernement (MEF et GTZ, 2003). Les revenus monétaires agricoles de la Région de l'Est du Burkina Faso sont pourvus à 56,3\% par les cultures de rentes, à savoir l'arachide et le coton (DRED-Est, 2003).

A l'instar de plusieurs pays africains, le Burkina Faso est confronté à une dégradation de son écosystème dont l'action de l'Homme constitue l'une des grandes causes (INERA, 2000 ; MECV, 2004 ; Simonsson, 2005). Selon Spack (1997), la forte dégradation des ressources naturelles dans la région de l'Est est liée à trois principaux facteurs dont les techniques culturales. Ainsi, l'adoption de bonnes pratiques agricoles peut constituer une garantie pour l'exploitation durable des ressources naturelles. A cet effet, les pratiques paysannes et les perceptions endogènes des problèmes environnementaux sont déterminantes dans l'élaboration des solutions aux problèmes de gestion de l'environnement. Des études ont abordé la problématique de la dégradation des ressources naturelles dans les agrosystèmes tropicaux (Taonda et al., 1995; Ouattara, 2006; Abba et al., 2007; Ranaivomanana, 2008 ; Traoré et Toé, 2008; Ouédraogo et al., 2009). L'étude effectuée par Abba et al. (2006) a révélé que l'extension de la culture de coton dans les régions de l'Ouest et du Centre de l'Afrique a entraîné des conséquences négatives pour l'environnement: changements climatiques, baisse de la diversité biologique et accélération de la dégradation des terres. Au Burkina Faso, en raison de la dégradation des conditions environnementales et de l'épuisement des sols, les zones cotonnières se sont déplacées de la partie située au nord des régions centrales vers le sud et le sud-ouest du pays (Hussein et al., 2005).

L'objectif visé par cette étude était de décrire les pratiques agricoles et de s'imprégner des perceptions paysannes des problèmes environnementaux dans l'optique d'accompagner les mécanismes décisionnels en matière de gestion durable de l'écosystème dans la province de la Kompienga.

\section{Matériel et méthodes}

\subsection{Zone d'étude}

Située à l'Est du Burkina Faso, la province de la Kompienga (Fig. 1) selon le dernier découpage administratif en 2000 comprend 3 départements qui sont: Pama (Chef lieu de la province), Madjoari et Kompienga. Cette province s'étend sur une superficie de $6998 \mathrm{~km}^{2}$ dont environ 1/ 3 est occupée par les réserves forestières et environ 1/3(223 000 ha) de réserves de faune. Le climat dans cette région est de type soudanien au sud et sahélien au nord (INERA, 2000). La moyenne des précipitations annuelles enregistrées de 1998 à 2007 dans la ville de Pama est 901,87 $\pm 180,23 \mathrm{~mm}$. 


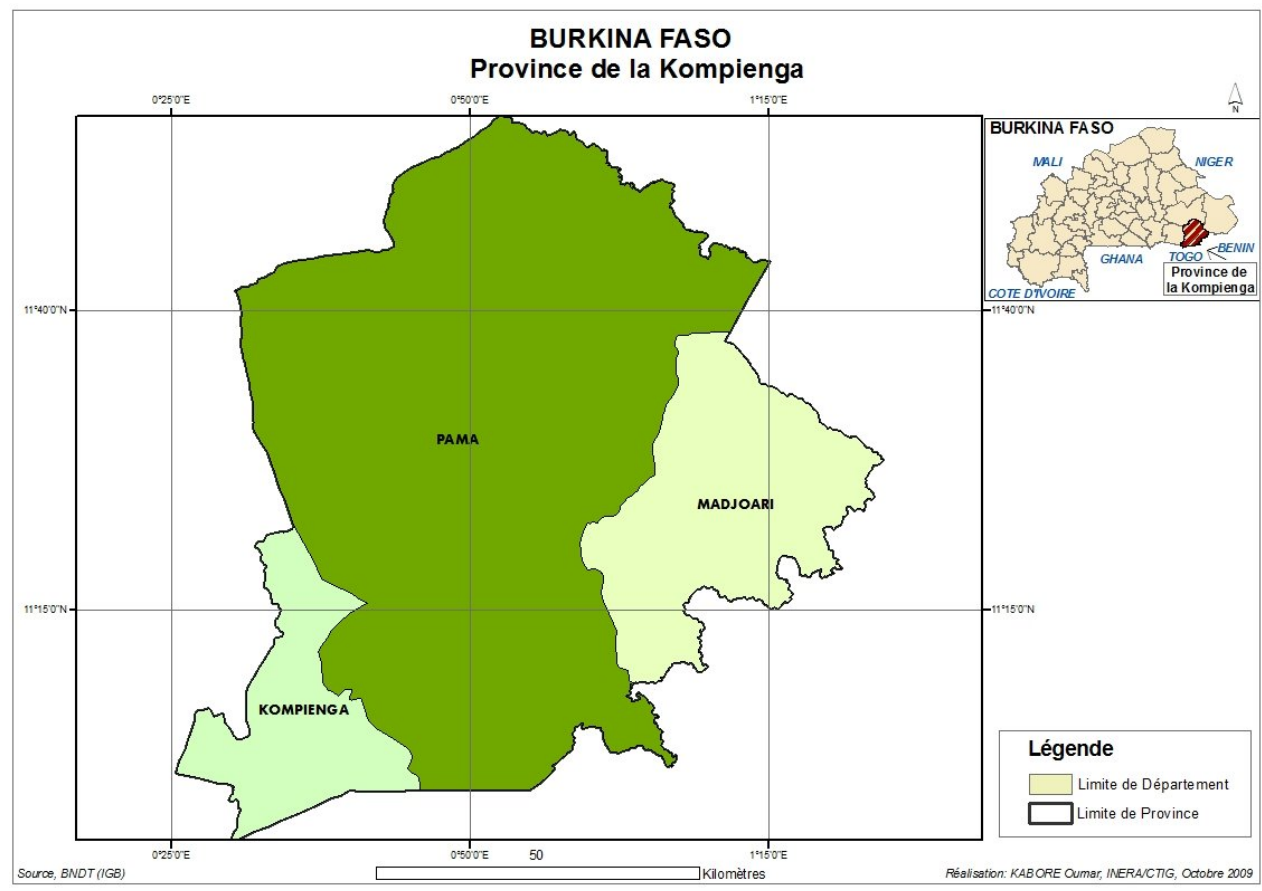

Figure 1 : Carte de la zone d'étude

\subsection{Collecte et analyse des données}

\subsubsection{Choix des acteurs cibles}

La méthodologie mise en œuvre dans la collecte des données de cette étude est basée sur une approche holistique qui a pris en compte les acteurs impliqués dans la gestion et/ou qui exploitent les ressources naturelles et par conséquent concernés par sa préservation. Un échantillon aléatoire de 60 UPC (Unité de Production Cotonnière) appartenant à 37 GPC (Groupement de Producteurs de Coton) des 3 départements de la province a été réalisé. Quant aux autres acteurs à savoir les membres de comité villageois de gestion de forêt (23), les apiculteurs (15), les pêcheurs (29), les maraichers (12) et les éleveurs (54) ; ils ont été choisis en fonction de leur disponibilité et de leur répartition géographique dans la province.

Deux méthodologies ont par ailleurs été retenues pour la collecte des données: (i) un questionnaire individuel et ouvert a été remis aux chefs des UPC après un test pour une meilleure adaptation aux réalités de terrain ; (ii) une interview semi structurée avec un focus-groupe a été appliquée aux autres producteurs (éleveurs, pêcheurs, apiculteurs, maraîchers et aux membres des comités villageois de gestion des forêts). Les axes essentiels de l'entretien ont été préalablement répertoriés dans un guide d'entretien (check-list). L'enquête s'est déroulée entre le mois d'août et octobre 2006. Un deuxième passage entre juillet et août 2007 sur les sites de production a permis la collecte de données et de faire des observations complémentaires.

\subsubsection{Paramètres considérés}

Les paramètres pris en compte dans l'enquête ont varié selon les acteurs cibles. Pour les producteurs de coton, il s'est agi de décrire les systèmes de production (équipements agricoles, les rotations culturales pratiquées, le niveau d'intégration de l'agroforestérie), les stratégies de gestion des ressources naturelles (l'usage des fertilisants organiques, la pratique de la jachère et les modes de préparation des sols) et d'avoir leur perception sur les impacts de la culture de coton sur l'écosystème.

Pour les autres acteurs cibles (éleveurs, pêcheurs, apiculteurs, maraichers et comité villageois de gestion de forêt), il s'est agi de faire 
un diagnostic des impacts négatifs de la cotonculture sur les activités de chaque catégorie socioprofessionnelle.

\subsubsection{Analyse statistique des données}

Les données collectées ont fait l'objet d'un dépouillement manuel et ont été saisies et analysées à l'aide du tableur EXCEL 2003. Les résultats obtenus ont été traités par des statistiques descriptives par détermination des moyennes, fréquences et écart-type.

\section{Résultats}

\subsection{Unité de Production Cotonnière (UPC) et production cotonnière dans la zone}

La culture de coton dans la province de la Kompienga est assez récente pour la majorité des producteurs (Fig. 2). L'ancienneté moyenne des producteurs de coton est de 4 ans pour l'effectif interviewé. Sur l'ensemble de l'effectif concerné par l'enquête, $58,33 \%$ des exploitations n'excède pas 3 ans d'ancienneté.

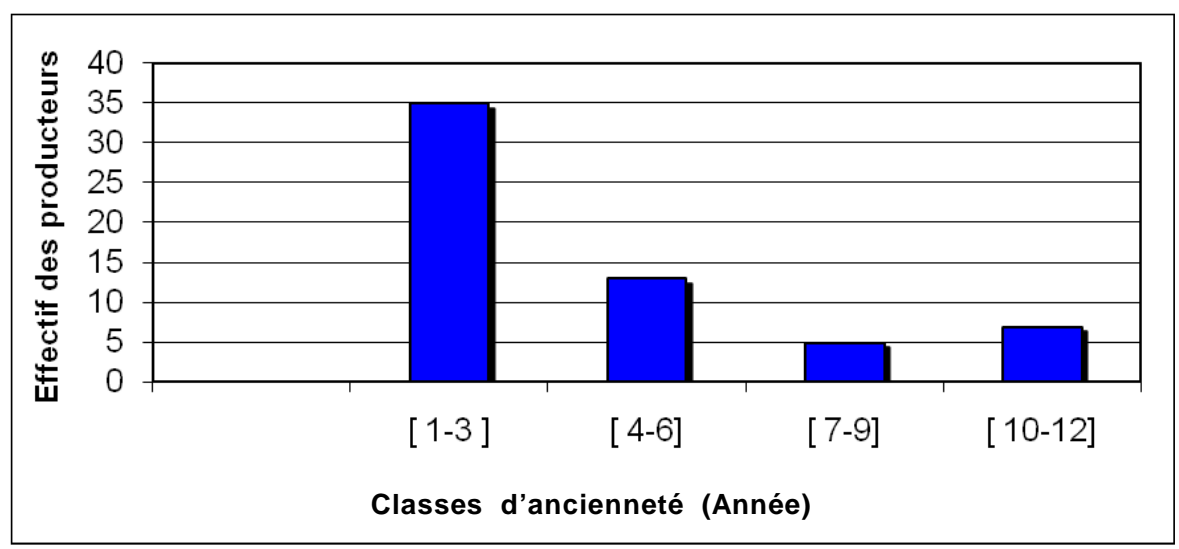

Figure 2 : Répartition des producteurs selon leur ancienneté dans la cotonculture

Des visites de terrain ont permis de répertorier dans la zone d'étude 145 Groupements de Producteurs de Coton (GPC). La production et les superficies emblavées de l'ensemble de la région cotonnière (zone de l'Est) ont connu un accroissement de 1996 à 2006 (Fig. 3 et Fig. 4). Par contre les rendements sont restés stationnaires et connaissent une baisse depuis 2004 avec un rendement moyen de 1054,33 tonnes/ha entre 1995 à 2006 dans la province de la Kompienga. Les caractéristiques des Unités de Production Cotonnière (UPC) de la zone sont décrites dans le tableau 1.

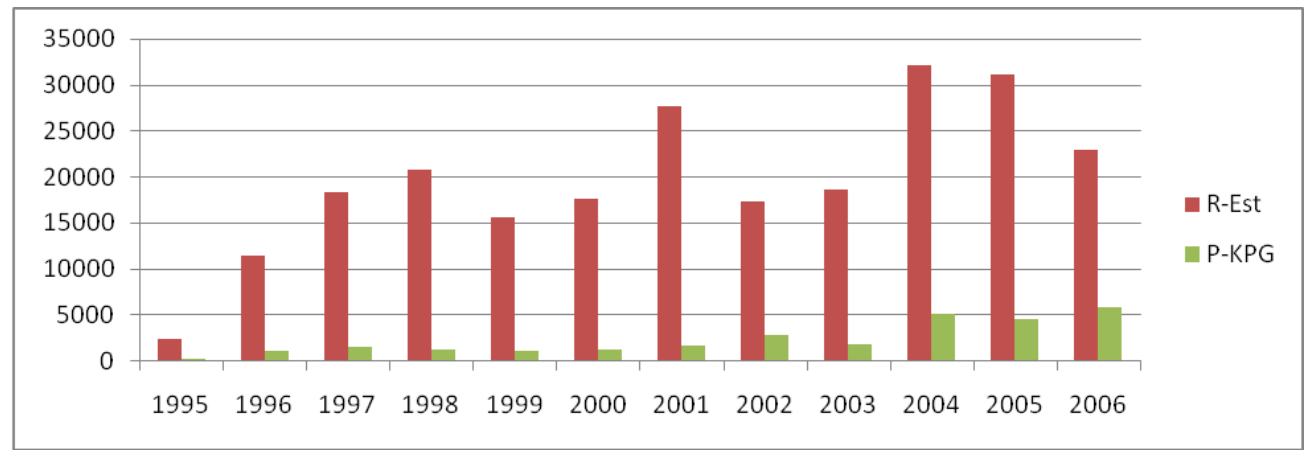

Figure 3 : Evolution de la production cotonnière dans la région de l'Est(R-Est) et dans la province de la Kompienga (P-KPG)

Source : Adapté de MAHRH (2007) 


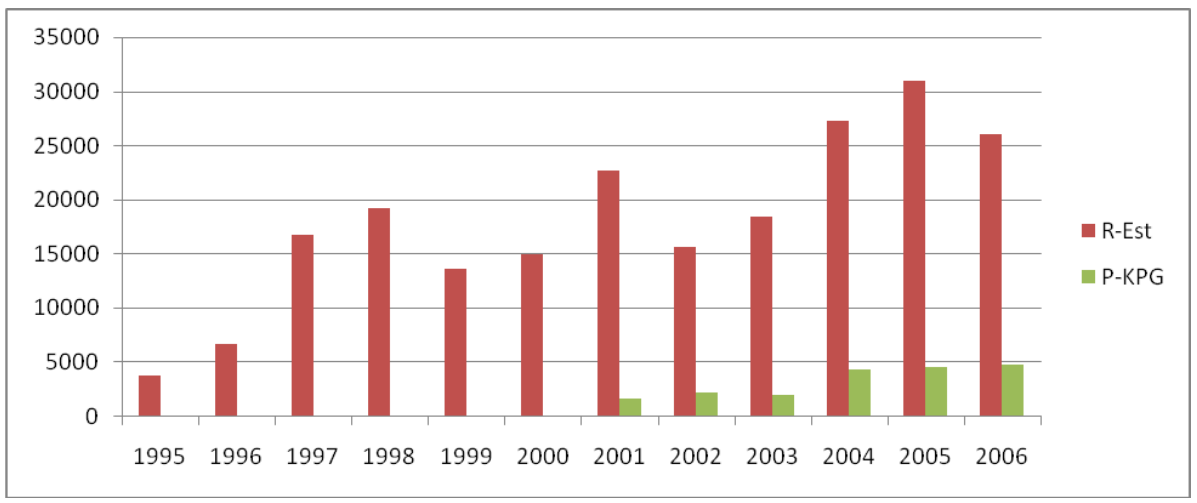

Figure 4 : Evolution spatiale des superficies emblavées dans la région de l'Est (R-Est) et dans la province de la Kompienga (P-KPG).

Source : Adapté de MAHRH (2007)

Tableau 1 : Caractéristiques des Unités de Production de Coton (UPC) dans la province de la Kompienga

\begin{tabular}{lc}
\hline variables & Valeur ou Proportion \\
\hline Nombre de producteurs & 60 \\
Ancienneté de $1-3$ ans (\%) & 58 \\
Ancienneté de 4-6 ans (\%) & 22 \\
Ancienneté de plus de 6 -10 ans (\%) & 20 \\
Surface emblavée en coton (ha) & $3,31 \pm 2,60$ \\
Surface emblavée en céréales (ha) & $4,94 \pm 3,63$ \\
Producteurs Mossi (\%) & 48 \\
Producteurs Gourmantchés (\%) & 38 \\
Autres Ethnies* (\%) & 14 \\
Producteurs à l'âge $>20<31$ ans (\%) & 35 \\
Producteurs à l'âge $\geq 31<42$ ans (\%) & 33,33 \\
Producteurs à l'âge $\geq 42 \leq 65$ ans (\%) & 31,67 \\
Producteurs non instruits (\%) & 25 \\
Producteurs alphabétisés (\%) & 30 \\
Producteurs de l'école classique (\%) & 11 \\
Producteurs de l'école coranique (\%) & 34 \\
Producteurs de profession agriculteur (\%) & 88,33 \\
Producteurs d'autres professions (\%) & 11,67 \\
\hline
\end{tabular}

$\left(^{*}\right)=$ Bissa, Bariba, Djerma, Tamarba et Peulh

\subsection{Systèmes de production}

\subsubsection{Equipements agricoles}

L'enquête a révélé que les équipements agricoles motorisés sont quasiment inexistants. Par contre $76,67 \%$ des producteurs détiennent au moins une charrue bovine ou asine. Ces équipements agricoles sont importants dans les exploitations cotonnières. Pour effectuer les traitements phytosanitaires du cotonnier, $68,88 \%$ de l'échantillon enquêté dispose au moins d'un pulvérisateur. D'autres équipements comme les rayonneurs, les semoirs et les charrettes sont rencontrés fréquemment chez les producteurs.
En outre, les producteurs possèdent des animaux (bovins, ovins, caprins et asins) pour les activités agricoles.

\subsubsection{Modes de préparation des sols}

Dans la préparation des sols du cotonnier trois modes sont employés pour le débroussement. II y a l'abattage des arbres et arbustes, la mise à feux (Photo 1) de la végétation et l'usage d'herbicides totaux pour le désherbage. Il s'en suit en général pour l'un ou l'autre des modes employés, une opération de labour des champs afin de procéder aux semis. 


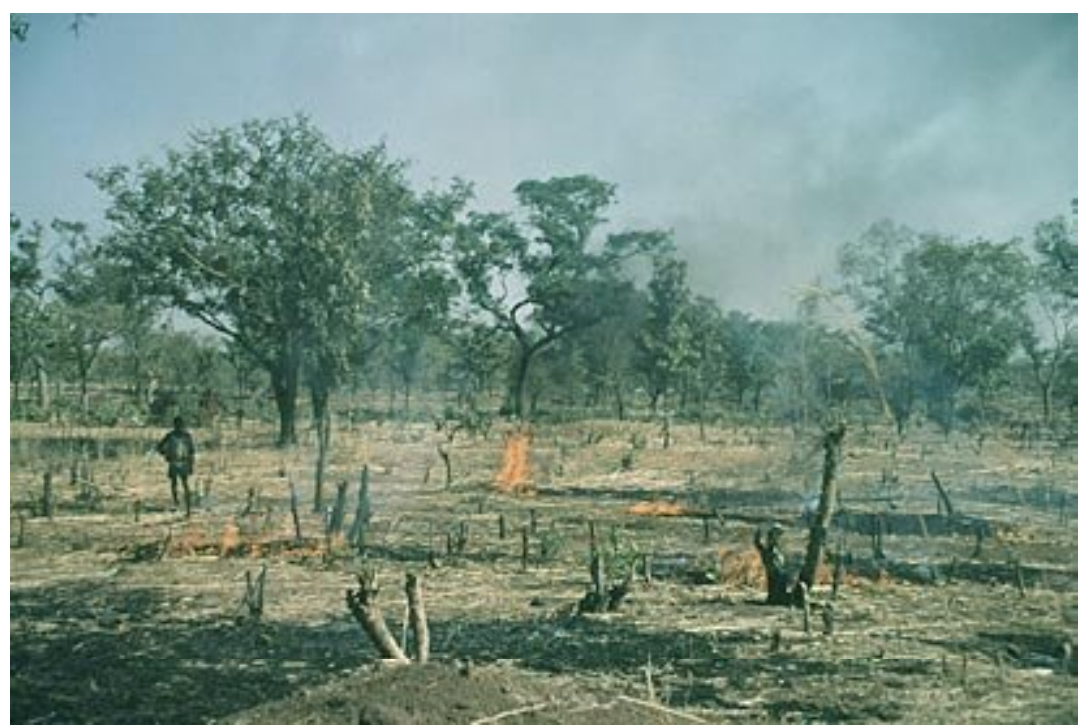

Photo1: Défrichement par brûlis pour une plantation de coton

\subsubsection{Rotations culturales}

Dans la zone d'étude, la rotation culturale est très courante. Près de $94 \%$ des producteurs enquêtés affirment la pratiquer. Elle concerne la plupart des cultures. L'ordre de rotation vise à permettre aux cultures de bénéficier des arrières effets des fertilisants chimiques (l'urée et le NPK) et de lutter contre certaines adventices. Le tableau 2 relate les différents types de successions culturales pratiquées par les producteurs dans la zone. II en ressort que l'ordre de succession "coton-céréale-coton » est le plus fréquent $(63 \%)$.

Tableau 2 : Successions culturales pratiquées dans la zone de l'étude

\begin{tabular}{lcc}
\hline Ordres de successions & Effectif & $\begin{array}{c}\text { Proportion des } \\
\text { UPC(\%) }\end{array}$ \\
\hline Coton-maïs-coton & 15 & 37,5 \\
Coton-maïs-sorgho-coton & 5 & 12,5 \\
Coton-sorgho-coton & 3 & 7,5 \\
Mil-coton-maïs-mil & 2 & 5 \\
Sorgho-coton-sorgho & 2 & 5 \\
Coton-petit mil-coton & 2 & 5 \\
Haricot-coton-maïs-haricot & 1 & 2 \\
Coton-maïs-pastèque-coton & 1 & 2,5 \\
\hline
\end{tabular}

\subsection{Stratégies de gestion des ressources naturelles}

\subsubsection{Usage des fertilisants organiques}

L'étude a révélé que $55 \%$ des producteurs disposent de fosses fumières ou de compostières fonctionnelles. Les fosses qui existent ont des anciennetés diverses. Aussi, près de $80 \%$ des UPC possèdent des ruminants pour la réalisation des opérations de labour et de la fertilisation (apport du fumier) des champs de cotonniers. 


\subsubsection{Pratique de l'agroforesterie}

La pratique de l'agroforesterie, chez les cotonculteurs est très limitée, notamment chez les agriculteurs allochtones. La plupart des producteurs $(100 \%)$ sont persuadés de l'importance de cette pratique, mais en pratique, opèrent d'autres choix. La raison évoquée est la tenure du foncier. Selon les producteurs allochtones, il ne leur est pas recommandé de planter les arbres car les propriétaires terriens voient en cela une appropriation des parcelles d'exploitation. Nous avons constaté que dans les champs de coton, les arbres qui sont protégés sont généralement les essences fruitières comme le karité, le niébé, le raisinier et le tamarinier.

\subsubsection{Pratique de la jachère}

Dans la zone concernée par l'étude, cette technique n'est plus courante chez $70 \%$ de producteurs. Ils l'expliquent par le manque d'espace cultivable. Cette situation est valable non seulement chez les migrants mais aussi chez les autochtones. Les cotonculteurs qui pratiquent la jachère disent être conscients de son importance dans la réhabilitation des sols.

\subsection{Perceptions paysannes des impacts de la cotonculture sur l'environnement}

\subsubsection{Impact de la cotonculture sur le choix des spéculations agricoles vivrières}

Le choix des spéculations agricoles par les cotonculteurs et les changements qui y sont intervenus depuis 2005 et avant leur implication dans la culture de coton fournit des informations sur la stabilité des choix de production agricole dans la province de la Kompienga. II en ressort que des producteurs $(27 \%)$ n'ont pas opéré de changement. En d'autres termes, l'implication de ces producteurs dans la culture cotonnière n'a pas eu d'effet sur les autres spéculations traditionnellement exploitées. Le coton coexiste, de façon générale, avec les autres cultures. Toutefois, $35 \%$ des producteurs ont dû abandonner certaines cultures. Parmi celles-ci figurent l'arachide, le mil, le riz, le sorgho, l'igname, le niébé et le melon. En revanche, 38\% des producteurs ont introduit de nouvelles spéculations; le maïs a été ajouté par $37,04 \%$ des producteurs enquêtés.

\subsubsection{Problèmes environnementaux liés à l'usage des pesticides}

Près de $90 \%$ de producteurs de coton estiment que l'utilisation des pesticides peut constituer un danger pour l'Homme par la contamination des eaux. Les producteurs de coton établissent la relation entre les risques de contamination des eaux et des poissons par les pesticides et les risques d'intoxication pour les êtres humains qui les consommeraient. Par contre, pour le sol, les réponses sont mitigées. Environ $48,3 \%$ des cotonculteurs pensent que l'application des pesticides peut baisser la fertilité des sols ; $10 \%$ de l'échantillon disent ne pas pouvoir se prononcer sur cette question et $25 \%$ des producteurs de coton estiment que les pesticides n'ont pas d'effets négatifs sur les sols. Selon eux, l'utilisation des pesticides contribuerait bien au contraire à renforcer et à maintenir sa fertilité.

Les problèmes environnementaux liés à la culture de coton dans la zone et rencontrés par les différents groupes d'acteurs sont consignés dans le tableau 3. Les apiculteurs rencontrés affirment que la baisse de leur récolte de miel est le fait de la rareté des abeilles sur leur site de production et cela est imputable aux pesticides utilisés en culture cotonnière. Ceux du village de Kpodjari imputent également la réduction des abeilles dans les ruches à l'utilisation des pesticides dans les champs de coton.

Quant aux éleveurs, pécheurs et maraîchers, ils affirment tous que la culture de coton dans la zone, notamment l'utilisation des pesticides et l'extension des superficies entravent sérieusement leurs activités. Des cas de conflits et d'animaux morts après avoir ingéré du fourrage pulvérisé par les insecticides ont été révélés lors des investigations dans les villages de Kabonga et de Kpodjari. 
Tableau 3 : Grille d'appréhension des problèmes environnementaux induits par la cotonculture par groupes d'acteurs.

\begin{tabular}{ll}
\hline Groupes d'acteurs & Problème environnemental identifié \\
\hline Eleveurs & -Difficile accès aux espaces de pâture \\
& - Contamination du fourrage vert par les pesticides \\
& -Intoxication des animaux \\
& -Contamination des eaux par les pesticides \\
\hline Apiculteurs & -Diminution de la population apicole \\
\hline Comité Villageois de & -Contamination du miel \\
Gestion de Forêt & -Empiètement des limites des réserves forestières \\
\hline Maraîchers & -Utilisation de pesticides non homologués \\
\hline & -Empiètement des berges du lac \\
Pêcheurs & -Contamination des eaux \\
\end{tabular}

Source: Données des interviews semi structurées (2006 et 2007)

\section{Discussion}

La caractérisation des exploitations laisse entrevoir à l'émergence de la culture du coton dans la zone d'étude. Les systèmes d'exploitation agricole sont décrits comme étant extensifs et fortement consommateurs des ressources naturelles (Spack, 1997; INERA, 2000). Les équipements agricoles motorisés sont quasiment absents. Cependant cette situation peut évoluer au fil du temps. C'est le constat fait dans la zone cotonnière de l'Ouest du pays par Bélem (1985). Cette évolution engendre des changements sur les systèmes de production avec un accroissement inéluctable des superficies exploitées chez les producteurs (Tersiguel, 1992 ; Spack, 1997 ; Abba et al, 2007). En effet, environ $11,60 \%$ des producteurs ont plus de 10 ans d'activité dans la cotonculture.

L'utilisation de la fumure organique est peu répandue chez les producteurs. Pourtant plusieurs travaux ont montré l'importance de l'apport de l'amendement organique dans le maintien de la qualité agronomique des sols (Hartemink et Bridges, 1995 ; INERA, 2000 ; Hibra-Samgue, 2004 ; FAO, 2007 ; Masto et al, 2008). De même l'étude de Savadogo et al. (2008) a montré que la dégradation des pesticides est plus élevée dans les sols amendés par la matière organique comparée aux sols non amendés.

L'augmentation des superficies de production (coton et autres céréales) a été exprimée par les cotonculteurs. Cela est une source de déforestation et ce processus peut entraîner la perte de biodiversité végétale et animale, la dégradation des sols et des eaux (Abba et al, 2006; FAO, 2007). Cette extension des superficies est négativement vécue par les éleveurs. Ils voient en cela une restriction des zones de pâture des animaux. Quant aux membres des comités villageois de gestion des forêts, l'extension des superficies est considérée comme le facteur qui contribue à la disparition des formations végétales et à l'empiètement des limites de réserves de forêt. En effet, la destruction de 
biotopes floristiques (végétales) et fauniques conduit à la disparition d'espèces végétales et aux migrations ou la disparition d'espèces animales, parmi lesquelles les insectes pollinisateurs telles que les abeilles, ce qui entraine la baisse de la productivité agricole et forestière. Aussi, la zone connaît une forte anthropisation autour des réserves forestières avec un développement d'activités agricoles, cela est dû à la présence du barrage de la Kompienga et des ressources naturelles relativement abondantes par rapport aux autres régions du pays. Selon Ranaivomanana (2008) et FAO (2007), l'emprise de l'homme sur l'espace se traduit par l'extension de l'habitat et les exploitations agricoles. En outre, Ouattara (2006) a mis en évidence que les zones mises en culture dans la Kompienga ont connu une augmentation d'environ $30 \%$ avec une régression des savanes boisée et arborée respectivement de $76,5 \%$ et $60 \%$. Par ailleurs, l'agroforesterie est rarement pratiquée dans les UPC et la pratique des feux est une option privilégiée de préparation des sols, ce qui constitue des risques écologiques. Dans de telles conditions, le renouvellement du stock organique par les feuilles des ligneux et par l'activité racinaire n'est pas assuré. Selon Zombré (2003) et Abba et al. (2006) la durabilité des systèmes de production dans les pays sahéliens ne peut être une réalité sans la présence des arbres dans les écosystèmes, eu égard à leur dynamique multifonctionnelle.

Les résultats ont montré un abandon de la jachère qui était la technique de restauration des sols. Cela est probablement dû à la forte démographie à laquelle est confrontée la zone suite aux migrations des populations venues de la région centrale et du nord du pays à la recherche des terres fertiles. Hibra-Samgue (2004) et Ouédraogo et al. (2009) ont établi une forte corrélation entre l'augmentation de la densité des populations et des effets négatifs induits sur les ressources naturelles. La pression sur les terres exploitables affecte leur fertilité ce qui se traduit par une baisse des rendements agricoles (Traoré et Toé, 2008). La conséquence de la pratique de la jachère des terres converties est le déplacement des activités agricoles vers les parcelles forestières (FAO, 2007). En somme, la jachère a un intérêt environnemental certain et peut devenir un outil de réaménagement de l'espace. Selon Le Clech (1998) la jachère comporte des fonctions agronomiques et sociales par la lutte contre les adventices, le stockage de l'eau, la réduction du parasitisme tellurique, la limitation de la pollution nitrique et de l'érosion dans les agrosystèmes où elle est pratiquée.

L'état des lieux de la perception des impacts environnementaux a mis en évidence les méconnaissances des cotonculteurs sur les risques majeurs. Sougnabé et al. (2009) ont également constaté que les paysans du cotonnier du Tchad n'ont pas une bonne

connaissance des matières actives des pesticides utilisées, des doses d'application et de leurs impacts sur la santé humaine. Cette situation contraste avec celle des cotonculteurs de Kobé Dani et Okéowo au Bénin qui perçoivent bien les impacts sur l'environnement notamment l'utilisation des produits chimiques agricoles (OBEPAP, 2006).

L'effet d'entraînement de la cotonculture sur les autres spéculations agricoles notamment l'introduction du maïs a été mise en évidence par $37 \%$ des cotonculteurs. Cela peut s'expliquer par la possibilité d'accès aux intrants (NPK, urée) obtenus à crédit pour le coton et qui sert également à la fertilisation du maïs. Cela suggèrerait également une relation entre la culture de coton et celle du maïs. Cette forte relation a été favorisée par le dispositif d'encadrement des producteurs de coton dans lequel les compagnies cotonnières font la promotion de la culture de maïs en vue de valoriser les arrières effets de la fertilisation du cotonnier (Schwartz, 1991; Ton, 2006; Somda et al. 2006).

Dans notre étude les apiculteurs lient la baisse de la production du miel à l'utilisation des pesticides en cotonculture dans leur zone. II justifie leur point de vue par le fait que la quantité de miel obtenue dans les ruches placées à proximité des champs de coton serait de plus en plus faible par rapport aux autres sites de leurs activités. II n'y a pas eu de protocoles expérimentaux pour vérifier cette affirmation, cependant si cette assertion s'avérait, c'est une activité socioprofessionnelle qui serait en péril eu égard au rôle important que jouent ces insectes dans la pollinisation. 


\section{Conclusion}

L'étude a permis de décrire les pratiques agricoles et les perceptions paysannes des problèmes environnementaux dans la région cotonnière de l'Est du Burkina Faso. Une analyse de ces systèmes et modes de production dans les UPC en relation avec l'exploitation durable des ressources naturelles a permis d'en déduire les impacts susceptibles de mettre en péril l'équilibre de l'écosystème de cette région. La pression foncière et ses effets induits, l'absence de stratégies durables de renforcement des supports de production (amendement des sols, agroforesterie etc.) et la non perception des risques environnementaux liés à l'intensification agricole constituent des points de réflexion à considérer dans les plans de développement afin de permettre une meilleure intégration de toutes les activités agricoles dans cette zone.

C'est pourquoi des protocoles expérimentaux doivent être élaborés afin de cibler certains compartiments écologiques qui pourraient subir des dommages de la cotonculture. II s'agit de la population apicole, des eaux, des sols et des poissons.

Pour se faire, il est impératif pour les acteurs de la filière cotonnière d'adopter des mesures respectueuses de l'environnement pour une exploitable durable des ressources naturelles et de poser des axes de réflexion pour une cotonculture qui s'inscrive dans la prise en compte de la préservation de l'écosystème de la zone.

\section{Références citées}

Abba A. A., Hofs J. L., \& Mergeai G., 2007. Relever les défis environnementaux pour les filières cotonnières d'Afrique de l'Ouest et du Centre. Biotechnol. Agron. Soc. Environ. 10(3): 351-359.

Belem C., 1985. Coton et système de production dans l'Ouest au Burkina Faso. Thèse de 3 ème cycle en géographie de l'aménagement. Université de Montpellier III, France, 250pp.

CEDEAO-CSAO/OCDE, 2006. Le coton. Atlas de l'intégration régionale en Afrique régionale, Série économie, $20 \mathrm{p}$.

DRED-Est, 2003. Cadre régional de lutte contre la pauvreté. Direction régionale de l'économie et du développement de l'Est, Burkina Faso, 133p.

FAO, 2007. Interaction entre agriculture et forêt. $20^{\text {ème }}$ session, Comité de l'agriculture, Rome, 25-28 avril 2007.4p.

Hartemink A. E., \& Bridges E. M., 1995. The influence of parent material on soil fertility degradation in the coastal plain of Tanzania. Land Degradation \& rehabilitation, 6(4): 215-221.

Hibra-Samgue V., 2004. Gestion durable de la fertilité des sols sahéliens: stratégies adaptatives des paysans du plateau central du Burkina Faso face à la variabilité climatique. Cas de la province du Zandoma. Mémoire de DESA, Université Abdou Moumini, Niger, 79pp.

Hussein K., Perret C., \& Hitimana L., 2005. Importance économique et sociale du coton en Afrique de l'Ouest : rôle du coton dans le développement, le commerce et les moyens d'existence. OCDE SAH/D 556, $71 \mathrm{p}$.

INERA., 2000. Bilan de 10 années de recherches 1988-1998. Document MESSRS/CNRST/ Burkina Faso, édition CTA. 115p.

Le Clech B., 1998. Agriculture et environnement. 2éme édition, éditions SYNTHESE AGRICOLE. Paris, France. 341p.

MAHRH (Ministère de l'Agriculture de l'Hydraulique et des Ressources Halieutiques), 2006. Résultats définitifs de la campagne agricole 2005/2006. Direction des statistiques agricoles. Ouagadougou, Burkina Faso, 58p.

MAHRH (Ministère de l'Agriculture de l'Hydraulique et des Ressources Halieutiques), 2007. Résultats définitifs des campagnes agricoles 1995/1996 à 2006/ 2007. Direction des statistiques agricoles. Ouagadougou, Burkina Faso, 67p.

Masto R. E., Chonkar P. K., Purakayaska T. J., Patra A. K., \& Singh D., 2008. Soil quality indices for evaluation of long-term land use and soil management practices in semi-arid sub-tropical India. Land Degrad. Develop. 19(5): 516-529.

MECV, 2004. Rapport sur l'état de l'environnement au Burkina Faso. SP/CONAGESE, Ouagadougou, Burkina Faso. 174p. 
MEF \& GTZ, 2003. Application des micros simulations. Etude de l'impact des variations du prix du coton sur la pauvreté rurale au Burkina Faso. Ministère de l'économie et des finances, Ouagadougou, Burkina Faso. 41p.

OBEPAB (Organisation Béninoise pour la Promotion de l'Agriculture Biologique), 2006. Identification des problèmes sanitaires et environnementaux liés aux polluants organiques persistants (Pops). Rapport d'étude, OBEPAB, Cotonou, Bénin, 42p.

Ouattara A., 2006. Mobilité spatiale de la population: nécessité de développement et risques de dégradation de l'environnement dans l'Est et le Sud-ouest du Burkina Faso. INSS, Ouagadougou, Burkina Faso, 35p.

Ouédraogo I., Savadogo P., Tigabu M., Cole R., Oden P.C. \& Ouadba J.M., 2009. Is rural migration a threat to environmental sustainability in southern Burkina Faso? Land Degrad. and Develop. 20(2): 217-230.

Ranaivomanana L.N.J., 2008. Identification des conditions d'appropriation de la gestion durable des ressources naturelles et des écosystèmes : Cas du grand récif de Toliara. Thèse de doctorat, ENSA de Renne, France, 199pp.

Savadogo P. W., Lompo F., Bonzi-Coulibaly Y L., Traoré S. A., \& Sedogo P. M., 2008. Influence de la température et des apports de matière organique sur la dégradation de l'endosulfan dans trois types de sols de la zone cotonnière du Burkina Faso. J. Soc. Ouest-Af. Chim. 26: 79-87.

Schwartz A., 1991. L'exploitation agricole de l'aire cotonnière burkinabé : caractéristiques sociologique, démographique et économique. Document de travail. ORSTOM. Ouagadougou, Burkina Faso.88p.

Simonsson L., 2005. Profil de vulnérabilité du Burkina Faso. Rapport d'étude de Stockholm environment institute, 38p.
Somda J., \& Nianogo A.J., 2006. Le coton et la perspective d'une gestion participative et durable des ressources naturelles dans la région Est du Burkina Faso. Communication, atelier sur la capitalisation des initiatives participatives de gestion des ressources naturelles dans l'Est du Burkina Faso. $1^{\mathrm{er}}-2$ Juin 2006. Fada N'Gourma (Burkina Faso), 13p.

Sougnabé S.P., Yandia A., Acheleke J., Brevault T., Vaissayre M., \& Ngartoubam L.T., 2009. Pratiques phytosanitaires paysannes dans les savanes d'Afrique Centrale. Actes du colloque "Savanes africaines en développement : innover pour durer », 2023 avril 2009. Garoua (Cameroun).13p.

Spack S., 1997. Stratégie de gestion durable des terroirs villageois de la région de l'Est, Burkina Faso, Géo-Regards, 31.147p.

Taonda S. J. B., Bertrand R, Dickey J., Morel J. L., \& Sanon K., 1995. Dégradation des sols en agriculture minière au Burkina Faso. Cahiers d'Agriculture; 4:363-369.

Tersiguel P., 1992. Boho-kari, village Bwa : Les effets de la mécanisation dans l'aire cotonnière au Burkina Faso. Thèse de Doctorat de Géographie. Université Paris X, France, $264 \mathrm{p}$.

Ton P., 2006. Promouvoir la production plus durable de coton : possibilités au Burkina Faso et au Mali. FAO/PNUE, Rapport final, Mars 2006. 71p.

Traoré K., \& Toé A. M., 2008. Capitalisation des initiatives sur les bonnes pratiques agricoles au Burkina Faso. Rapport de consultation, MAHRH/DVRD, Ouagadougou, Burkina Faso, $99 \mathrm{p}$.

Zombré N.P., 2003. Les sols très dégradés (Zipela) au centre nord du Burkina Faso: Dynamique, caractéristiques morpho-biopédologiques et impacts des techniques de restaurations. Thèse de doctorat. Université de Ouagadougou, Burkina Faso, 146 pp. 